\title{
A Unique Membrane Protein Is Expressed on Early Developing Limbic System Axons and Cortical Targets
}

\author{
Heather L. Horton and Pat Levitt \\ Department of Anatomy, The Medical College of Pennsylvania, Philadelphia, Pennsylvania 19129
}

The limbic system-associated membrane protein (LAMP) is a $64 \mathrm{kDa}$ protein that, in the adult brain, is present in cortical and subcortical regions comprising the limbic system (Levitt, 1984). The developmental expression of LAMP was studied in fetal rat brains to determine the specific patterns of distribution and the cellular elements that exhibit LAMP immunoreactivity. Light microscopic immunocytochemical analysis revealed that LAMP is expressed on neurons and their growing axons early in fetal development, at a time coincident with pathway formation and differentiation of limbic system nuclei. In the forebrain, where limbic system structures are heavily concentrated, immunoreactivity appears on subpopulations of axons in a temporal sequence that correlates with the time of formation of pathways carrying limbic system axons. Thus, staining is evident first at embryonic day (E) 15 on fibers within the internal capsule coursing from the diencephalon to cortex. LAMP immunoreactivity appears over the next $3 d$ in the anterior commissure, fornix, and corpus callosum. Ultrastructural immunocytochemical analysis reveals dense surface staining of fascicles of developing axons and growth cones. The axonal staining is transient, disappearing during the second postnatal week of development. In cerebral cortex, cells in presumptive limbic cortical regions such as lateral perirhinal sulcal cortex and prefrontal cortex are LAMP immunoreactive from the inception of the cortical plate. These cortical regions are clearly delineated from surrounding unstained nonlimbic areas as early as E15. Thus, LAMP expression in the cortex may represent one of the earliest markers of specific cytoarchitectonic areas. The early, stereotyped pattern of LAMP expression in subpopulations of developing axons and limbic regions, combined with our recent functional studies (Keller and Levitt, 1987), suggests that this unique cellsurface protein may be an important element in linking different components of a functional system in the mammalian brain.

\footnotetext{
Received Feb. 1, 1988; revised Apr. 8, 1988; accepted May 6, 1988.

We wish to thank Ms. Susan Fisher Hyland, Ms. Anna Zacco, and Dr. Andrea LaBorde for skilled technical assistance. We also thank our colleagues, Drs. Mary Barbe, Tim Cunningham, Michael Goldberger, Forrest Haun, and Marion Murray for their thoughtful comments. This research was supported by March of Dimes Basic Research Grant 1-919, NSF Grant BNS 8519647, and a fellowship from the Joseph and Esther Klingenstein Fund. Part of this work was performed while P. L. was a recipient of a National Downs Syndrome Society Scholar Award. H.L.H is a Medical Scientist Scholar of the Life and Health Insurance Medical Research Fund sponsored by the Lutheran Brotherhood.

Correspondence should be addressed to Dr. Pat Levitt, Department of Anatomy The Medical College of Pennsylvania, 3200 Henry Avenue, Philadelphia, PA 19129

Copyright (C) 1988 Society for Neuroscience $0270-6474 / 88 / 124653-09 \$ 02.00 / 0$
}

In the developing mammalian brain, different axon populations, within the same pathway, pass many potential targets but usually terminate to form synaptic contacts only within appropriate areas (Rakic, 1976; Loy et al., 1977; Crandall and Caviness, 1984). Cell-cell and cell-substrate interactions mediate the development of connections and probably involve a combination of mechanisms, including differential adhesion, diffusable neurotrophic factors, regulation of ion fluxes by specific channels, and mechanical cues (see Purves and Lichtman, 1985, for review). Specifically, it has been postulated that cell-surface macromolecules act as cues in the pathfinding and recognition events required to connect functional systems (Sperry, 1963; Singer et al., 1979; McKay et al., 1983; Bastiani et al., 1987), but evidence has been lacking for the early expression of these cell surface markers on functionally related subsets of axons and cellular regions in the vertebrate brain. A large number of membraneassociated macromolecules have been identified in the adult vertebrate CNS, but only a few of these are expressed in a restricted fashion on related groups of neurons (Trisler et al., 1981, 1986; Hockfield et al., 1983; Dodd et al., 1984; Levitt, 1984; Dodd and Jessell, 1985; Hendry et al., 1988). One, the limbic system-associated membrane protein (LAMP), is a 64 $\mathrm{kDa}$ glycoprotein that is found on the somata and dendrites of cortical and subcortical regions comprising the adult mammalian limbic system (Levitt,'1984; Levitt et al., 1986), which is involved in the mediation of emotional behavior (Papez, 1937), learning, and memory (Scoville and Milner, 1957; Penfield and Milner, 1958).

Because our anatomical studies in adult brain indicated that LAMP expression is associated with those areas forming limbic system circuits (Levitt, 1984; Levitt et al., 1986), we have attempted to determine whether the expression and spatial distribution of LAMP in developing brain occur at a time during development that corresponds to initial pathway formation and target recognition events. In addition, the immunocytochemical analysis was extended to the ultrastructural level to define the developing cellular components, such as axons, growth cones, or neuronal cell bodies, that express LAMP immunoreactivity. In the present study, utilizing a monoclonal antibody directed against LAMP in combination with light and electron microscopic immunocytochemical analysis, we report that LAMP is cxpressed during early fetal development on specific groups of growing axons and growth cones in pathways that carry limbic system axons. In addition to the presence of LAMP in basal forebrain and diencephalic limbic regions, cortical targets of some of these fiber groups express LAMP very early in fetal development. Cortical neurons exhibit great molecular heterogeneity, reflected by the large number of different neurotrans- 


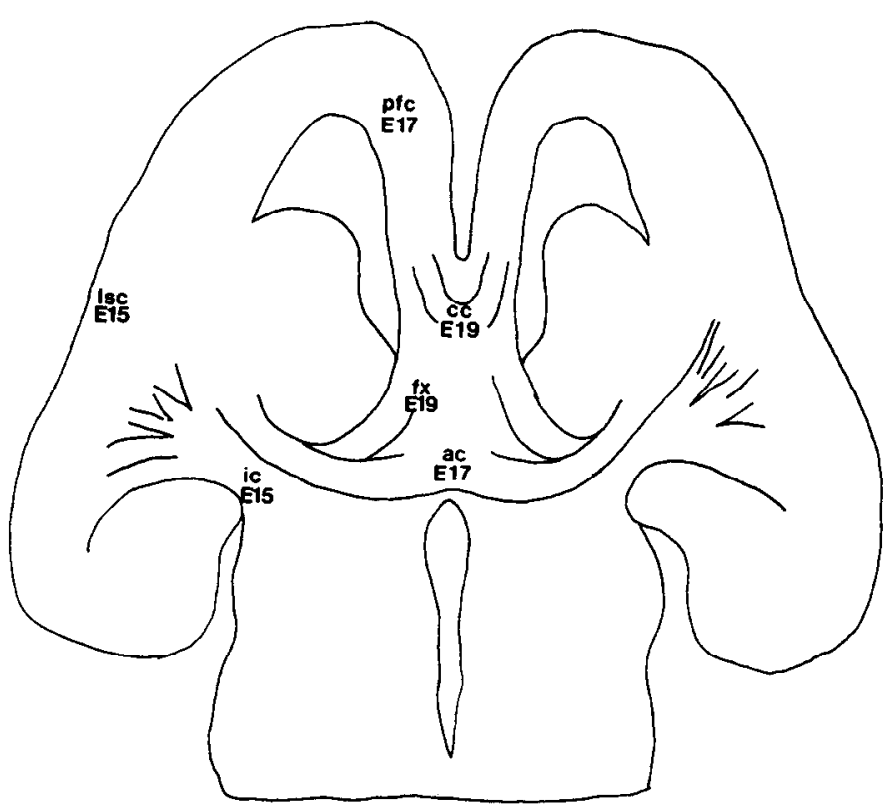

Figure 1. Diagram of a horizontal section through the developing rat forebrain summarizing the appearance of LAMP immunoreactivity during fetal life. The earliest detection of immunoreactivity is at E15 in the internal capsule $(i c)$, diencephalon $(d i)$ and lateral sulcal cortex $(l s c)$. Fetuses at E17 also contain stained fibers in the anterior commissure $(a c)$ and cclls in prefrontal cortcx $(p f c)$. By E1 8-19, LAMP-positivc fibcrs reach the midline in the corpus callosum $(c c)$ and are also evident in the entire extent of the fornix $(f x)$.

mitter pathways which represent subpopulations of neurons in the mature brain. Neighboring areas of cortex may have widely differing functions and anatomical connections, which may be parallelled by differing surface chemical identities. The presence of LAMP on a restricted population of early differentiating cortical neurons, shortly after the formation of the cortical plate, suggests that LAMP may represent one of the first molecular determinants of specific cortical areas.

\section{Materials and Methods}

Light microscopic immunocytochemistry. For the immunostaining, fetuses were obtained by cesarean section of timed-pregnant SpragueDawley albino rats (Holtzman Farms). The day of sperm-positive was designated as embryonic day (E) 0 . Fetuses were perfused with $4 \%$ paraformaldehyde in 0.1 м sodium phosphate buffer $(\mathrm{pH} \mathrm{7.2)}$, postfixed for $24 \mathrm{hr}$, and immersed in $20 \%$ sucrose $-0.1 \mathrm{M}$ sodium phosphate for $48 \mathrm{hr}$ at $4^{\circ} \mathrm{C}$. Immunolocalization of LAMP is sensitive to fixation; thus, we obtained inconsistent staining in immersion-fixed tissue. Immunocytochemical analysis was performed on rat fetuses beginning at E15, the earliest age at which we were able to perfuse fetuses with fixative transcardially. Whole heads were sectioned on a cryostat, and the sections were collected on chrome-alum-coated slides. The tissue was incubated in a 1:200-1:600 dilution of the anti-LAMP monoclonal antibody (Levitt, 1984) overnight at room temperature. Following extensive washing, sections were incubated in HRP-conjugated anti-mouse IgG (Jackson Immunoreagents) for $1 \mathrm{hr}$, washed, and reacted with $3,3^{\prime}$ diaminobenzidine in an ammoniated phosphate buffer to enhance staining. For staining of all axon pathways, a monoclonal antibody (IgM isotype) directed against the 200 and $160 \mathrm{kDa}$ neurofilament proteins was used. This antibody was generated in our laboratory (Pawlak-Byczkowska and Levitt, 1985) and stains early-developing fiber tracts. Fetal brain tissue sections were incubated overnight with a 1:50 dilution of antibody concentrated from culture supernatants by sodium sulfate precipitation. Following extensive washing in PBS, the sections were incubated for $30 \mathrm{~min}$ in a 1:30 dilution of rhodamine-conjugated goat anti mouse IgM (Jackson Immunoreagents). Sections were washed and coverslipped with a PBS-glycerol buffer containing $p$-phenylenediamine to retard fluorochrome fading (Platt and Michael, 1983). Immunoperoxidase-stained material was photographed on Kodak Technical Pan 4125 with a $470 \mathrm{~nm}$ filter to enhance contrast. Immunofluorescent material was photographed on the same film type using a conventional rhodamine excitation and emission filter combination.

Ultrastructural immunocytochemistry. Vibratome sections from animals perfused with $2 \%$ paraformaldehyde, $0.1 \%$ glutaraldehyde were incubated for $48 \mathrm{hr}$ at $4^{\circ} \mathrm{C}$ in a 1:1000 dilution of the anti-LAMP monoclonal antibody. The sections were washed for $1 \mathrm{hr}$, incubated for $2 \mathrm{hr}$ with a 1:100 dilution of HRP-conjugated goat anti-mouse IgG, washed extensively, and reacted as described above. Stained vibratome sections were osmicated, dehydrated, and flat-embedded in Embed 812 (Polaron). Regions of interest were dissected under an operating microscope and mounted onto plastic blocks for thin sectioning. Approximately $700-900 \AA$ thin sections were collected on 150 mesh copper grids for examination on a Jeol $100 \mathrm{CX}$ electron microscope. The sections were not counterstained with either uranyl acetate or lead citrate to facilitate visualization of the immunoperoxidase reaction product.

Immunodot assay. A modification of the dot-blot assay of Hawkes et al. (1982) was used to determine the presence of LAMP in fresh tissue samples. Hippocampus, fimbria/fornix, and a rostral segment of the corpus callosum were dissected on ice from adult and postnatal day $(P)$ 10 rats. Tissue was homogenized in $1: 1$ (wt/vol) of $50 \mathrm{~mm}$ Tris, $0.1 \mathrm{M}$ sodium phosphate buffer, pH 7.2 , containing 5 mM PMSF. Protein concentration was determined by the method of Lowry et al. (1951) and adjusted for each sample to equivalent levels. Five or $50 \mu \mathrm{g}$ of total protein was applied to nitrocellulose sheets (Schleicher and Schuell) using a slot-blot apparatus (Schleicher and Schuell). The samples were dried for several hours and the sheets blocked using $10 \%$ horse serum in PBS. The nitrocellulose strips were incubated in a 1:50 dilution of anti-LAMP overnight at $25^{\circ} \mathrm{C}$. Duplicate nitrocellulose strips containing the same samples were incubated in an identical protein concentration of the control antibody, an IgG2a isotype (same as the anti-LAMP) produced by a myeloma cell line (TIB-10, obtained from American Type Culture). Following extensive washing in the blocking solution, the strips were incubated in a 1:250 dilution of HRP-conjugated rabbit anti-mouse IgG (Accurate Scientific) and washed again, and the immunoreactivity was visualized using 4-chloronaphthol as the chromogen. Three separate experiments were performed.

\section{Results}

The immunocytochemical analysis of LAMP immunoreactivity revealed that the protein is expressed relatively early in rat brain development, in those cortical and subcortical areas that express LAMP in the adult. These include prefrontal and perirhinal cortex, septum, ventral pallidum, anterior, mediodorsal and periventricular thalamic nuclei, most areas of the hypothalamus, amygdala, reticular formation, and autonomic brain-stem nuclei. The expression of LAMP in these cellular regions occurs in a complex temporal fashion and is the subject of another report $(H$. L. Horton and $P$. Levitt, unpublished observations). Our analysis in this study concentrates on the fetal rat forebrain, including telencephalon and diencephalon, where fiber pathways carrying limbic system axons and cellular regions classically defined as limbic are most heavily concentrated. LAMP immunoreactivity is present in specific forebrain regions, including both developing axon pathways carrying limbic axons and cortical and subcortical cellular regions (Figs. 1, 2). The staining of some axon tracts in the fetal forebrain is in marked contrast to the pattern in the adult, in which LAMP is distributed only on the neuronal somata and dendrites in limbic system areas. Axons are never immunoreactive in the adult, and this is supported by our immunodot assay data (see below).

During the period between E15 and E19, immunoreactive axons appear in parts of the internal capsule, anterior commissure, fornix, and corpus callosum. There is differential expression of LAMP that coincides with the approximate time of formation of each pathway. Most important, there is no staining in tracts that do not carry limbic axons (cf. optic tract in Fig. 

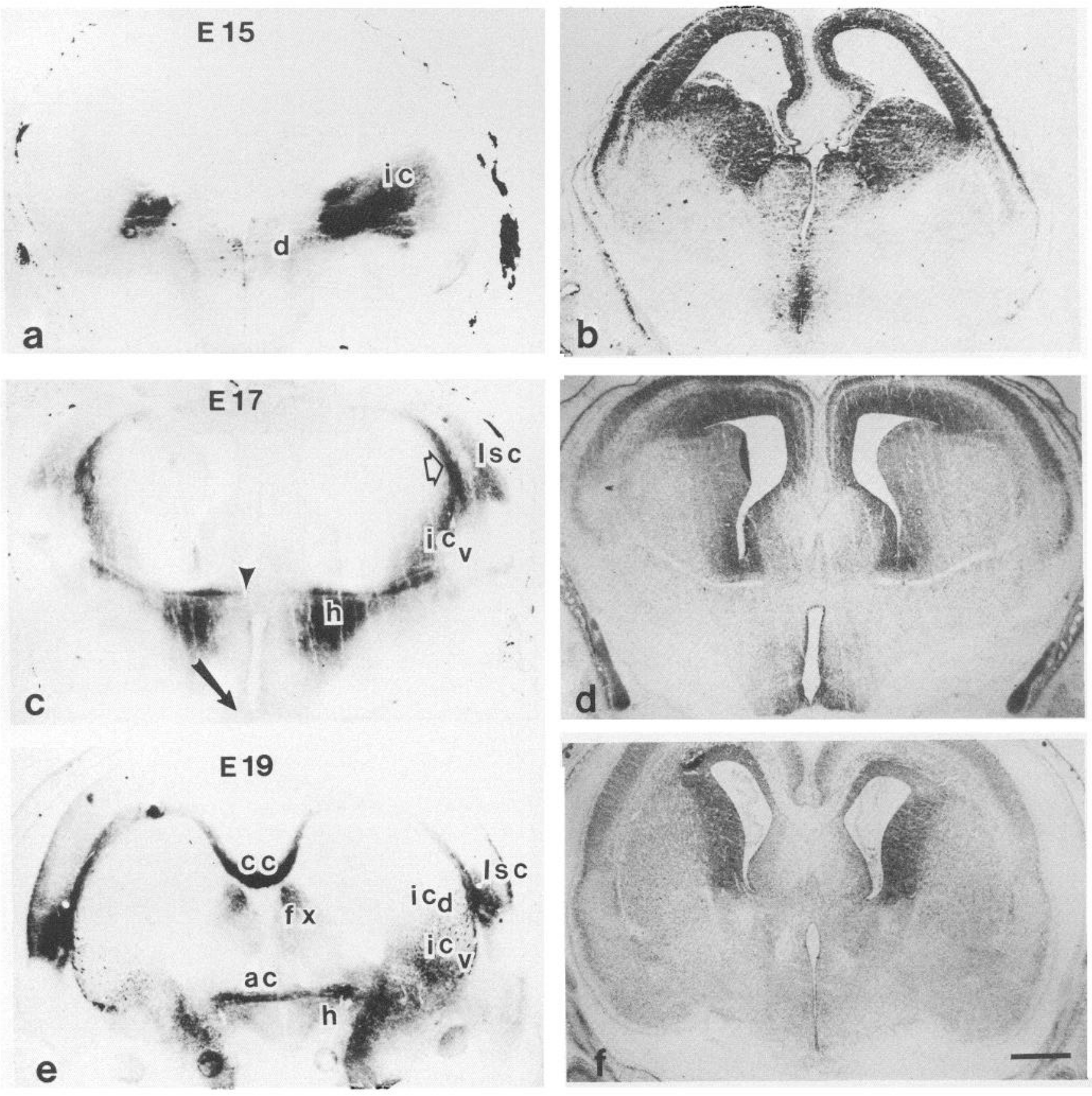

Figure 2. Comparison of LAMP immunoreactivity at E15 (a), $17(c)$, and $19(e)$ in a series of coronal sections. Nearly alternate sections stained with cresyl violet for each age are shown in $b, d$, and $f$. At E15, the internal capsule $(i c)$ exhibits very dense immunoreactivity. There is also light to moderate immunoreactivity in the diencephalon $(d)$. The staining in lateral sulcal cortex $(l s c)$ is not evident at this low magnification $($ see Fig. 6). At E17, immunoreactivity is now present in the ventral portion of the internal capsule (ic $c_{v}$ ), and in fibers that continue subcortically in the external capsule (open arrow). The staining in the hypothalamus $(h)$ and lateral sulcal cortex is also very dense at this age. Note that in the anterior commissure $(a c)$, LAMP-positive fibers are present in the tract but have not reached the midline (arrowhead). The optic tract does not express LAMP (arrow), nor do the fibers in the dorsal portion of the internal capsule $\left(i c_{d}\right)$. By E19, immunoreactivity is evident in each of the areas noted above and in the fornix $(f)$ and in fibers crossing in the corpus callosum $(c c)$. Scale bar for all figures, $500 \mu \mathrm{m}$.

2), and in those tracts that carry limbic axons among other presumably nonlimbic fibers (e.g., corpus callosum, internal capsule), only subsets of fibers are immunoreactive. For example, the earliest staining (E15) within the forebrain is found on subsets of axons coursing in the ventral portion of the internal capsule (Fig. 2). The intense immunoreactivity visualized in these fetuses at E15, the earliest age examined, suggests that the axons in the internal capsule may express LAMP prior to this age. Most fibers in the middle and dorsal part of this pathway are unstained at E15 and remain unstained throughout development (Fig. 2c-f). Selective expression of LAMP on subsets of internal capsule fibers can be demonstrated by the staining of serial 8- $\mu \mathrm{m}$-thick sections with anti-neurofilament (anti-NF) and anti-LAMP antibodies. All of the axon bundles running through the neostriatum are NF positive, but only certain subsets exhibit LAMP immunoreactivity (Fig. 3). The LAMP-stained ventral 
Figure 3. Immunohistochemical staining of neurofilament protein $(a)$ and LAMP $(b)$ in serial $8 \mu \mathrm{m}$ cryostat sections through the ventral aspect of the internal capsule at E17. Immunofluorescent neurofilament-positive axons are evident throughout the field. Several axon bundles that are double-labeled $(1,2)$ with both antibodies are present in the ventral part of the fiber tract. Some of the neurofilament-labeled (3) axons in the central part of the capsule are unlabeled in the section stained with the anti-LAMP antibody. Scale bar $(a$ and $b), 50 \mu \mathrm{m}$.
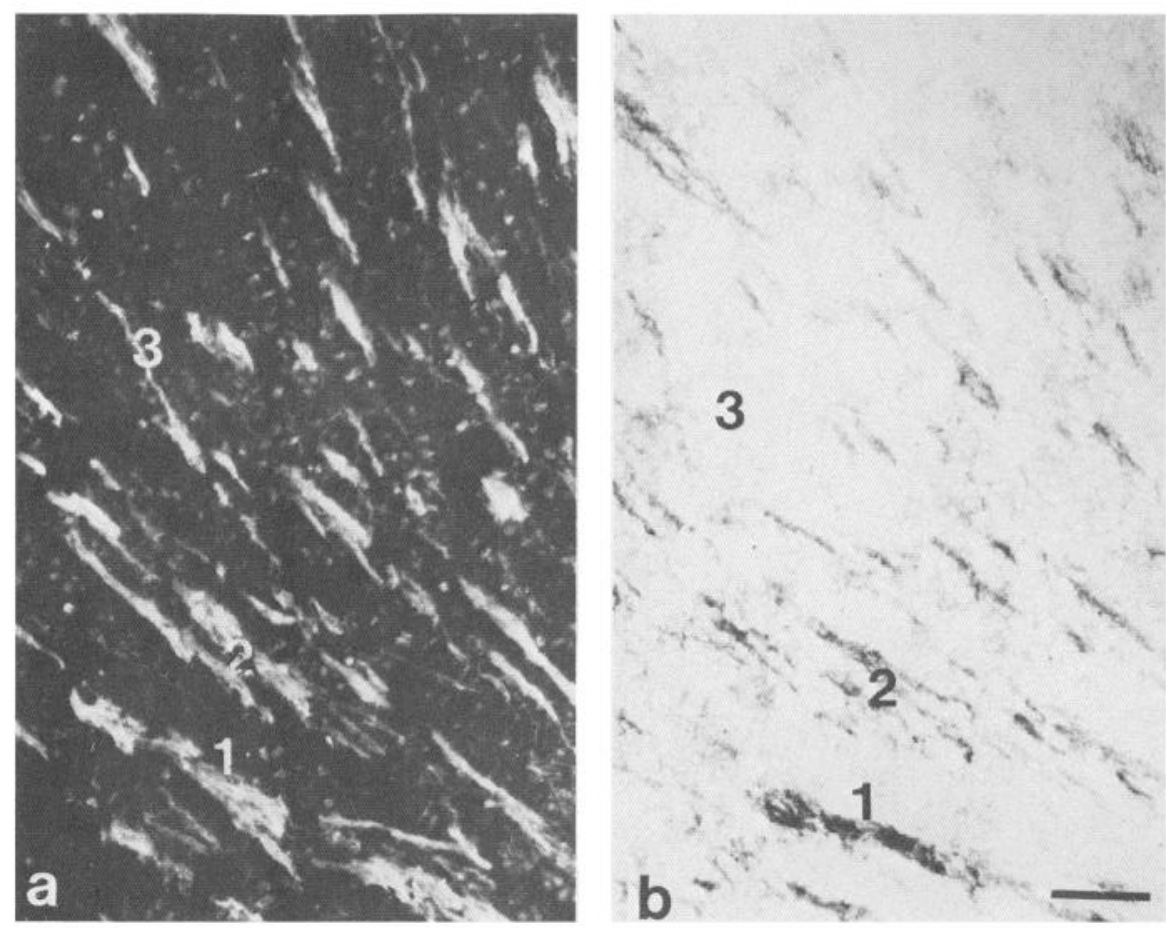

fibers can be traced at E15 from medial and ventral thalamic regions to lateral cortex just $24-36 \mathrm{hr}$ after the peak of neurogenesis in those parts of the thalamus that project to lateral sulcal cortex (Altman and Bayer, 1978). Some of the fibers continue in the external capsule, coursing dorsally in subcortical white matter towards the corpus callosum (Fig. $2 c-f$ ). There is no evidence of axons descending from LAMP-immunoreactive cortical regions into the capsule at E15, although we cannot eliminate the possibility that some LAMP-positive fibers originate in cortex at this age. In the developing anterior commissure, staining of serial sections with anti-NF and anti-LAMP show that while some NF-positive a kuns completely cross the midline at E17 (not shown), LAMP-immunoreactive fibers only reach part of the way towards the midline at this time (Fig. 2c) and then cross $24 \mathrm{hr}$ later. The corpus callosum, which forms rostrally 36-48 hr later (Valentino and Jones, 1982), contains subsets of LAMP-positive axons at the time of its formation. These can be seen first at E16-17 (Fig. 2), prior to the actual midline crossing of any axons. LAMP-positive axon bundles are among the first to cross at E18-19 (Fig. 2), along with NFpositive, non-LAMP immunoreactive axons.

Electron microscopic immunocytochemical examination confirms our light microscopic observations. We observed small fascicles of LAMP-immunoreactive axons that intermingle among unstained fascicles (Figs. 4a; 5, $a, b$ ). Patches of immunoreactivity are present at intervals on the axonal membrane along the length of longitudinally cut axon bundles (Fig. 5, $a$, $b$ ). At all fetal ages examined, the most dense immunoreactivity appears on the surface of growth cones and associated filopodia (Figs. $4 a ; 5, a, b$ ). We found no evidence of staining on agranular reticulum and cytoplasmic vesicles of the growth cones (Figs. $4 b ; 5, a, b)$.

LAMP immunoreactivity is also present on neurons in diencephalon and cortex by E15. LAMP-positive cells are evident in the cerebral cortex (see below) and in the diencephalon, dis- tributed throughout the hypothalamus and in classically defined limbic regions of the thalamus (Papez, 1937; Domesick, 1972; Wyss et al., 1979). By E17, LAMP staining is present in basal forebrain and septal nuclei. LAMP-immunoreactive cells are not detectable in any regions of the ventricular zone in the forebrain. At the first appearance of the cortical plate, however, LAMP-positive cells are evident in very specific cortical areas. Cortical cells in the intermediate zone, subplate, and deepest part of the cortical plate are the first to be immunostained at E15 (Fig. 6), following the classic inside-out gradient of cortical maturation (Hicks and D'Amato, 1968; Rakic, 1974). There is also a small amount of staining in the marginal zone at E15, representing either early-arriving afferents or early-generated superficial neurons. Differences in time of origin or maturity of neuronal populations cannot explain the differences in LAMP immunoreactivity between cortical areas, as previous studies in rodents have shown that cytoarchitectonically and functionally distinct neighboring regions of cerebral cortex have very similar times of origin (Hicks and D'Amato, 1968; Smart and Smart, 1982). This was confirmed in our examination of animals injected with ${ }^{3} \mathrm{H}$-thymidine on either E14, 15 , or 16 , revealing no difference in the laminar pattern of cell labeling between LAMPimmunoreactive sulcal cortex and the areas directly ventral (piriform) or dorsal (primary sensory) to this zone (unpublished data). Thus, LAMP expression clearly delineates prefrontal and lateral sulcal gustatory and visceral cortex at least within a few days of the genesis of the first neurons that comprise these areas. Of those molecules that appear to be unique to subpopulations of mature cortical neurons, LAMP is one of the earliest expressed, found at a time similar to the more ubiquitously distributed DARPP-32 (Foster et al., 1987) and occurring substantially earlier than the initial detection of most cortical neuropeptides and neurotransmitters (McDonald et al., 1982; Gall et al., 1984; Miller, 1986; Chun et al., 1987).

Immunocytochemical analysis of postnatal animals has dem- 

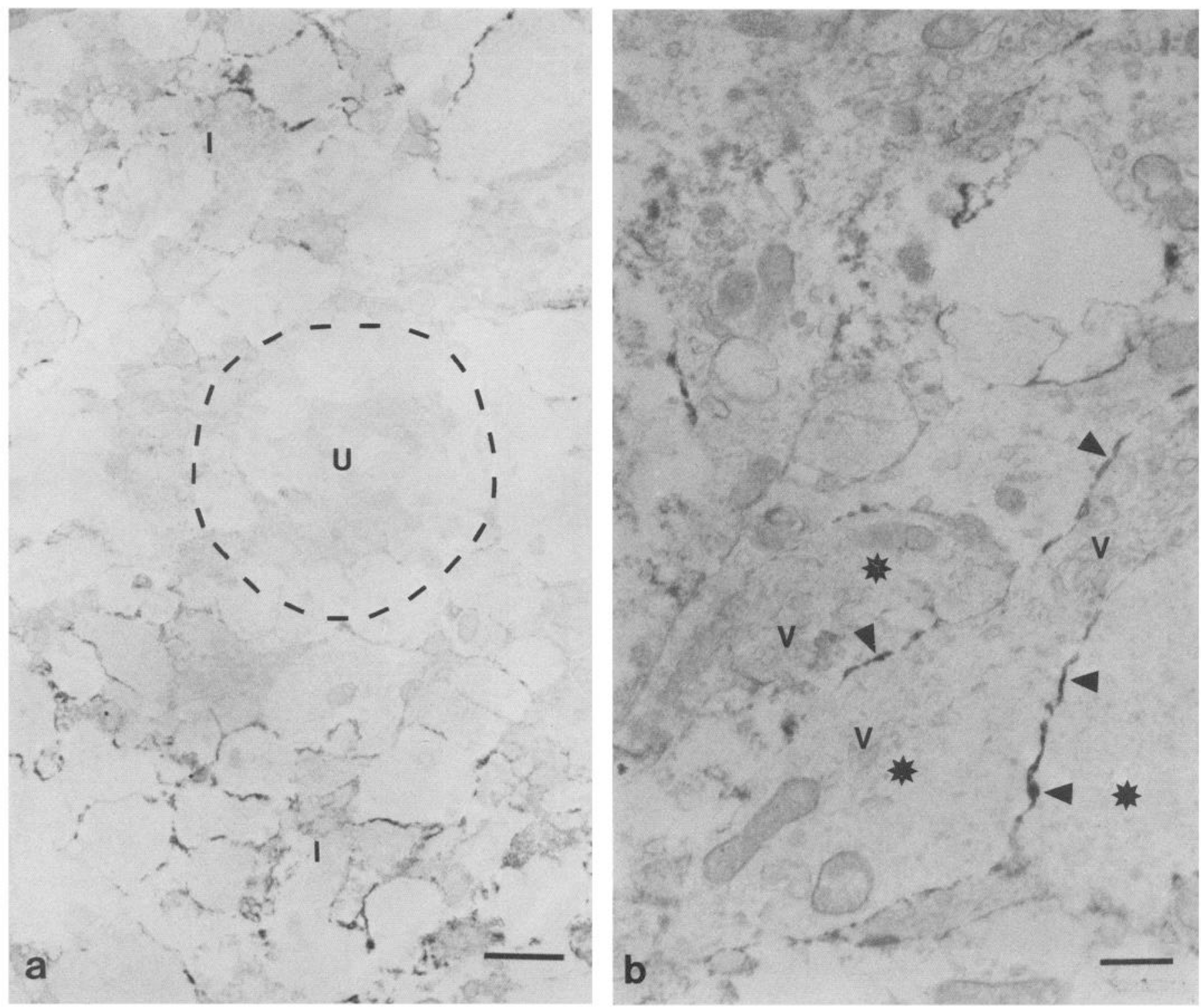

Figure 4. Electron microscopic immunocytochemical localization of LAMP in the ventral internal capsule at E19. In a cross section through the internal capsule (a), distinct groups of labeled, LAMP-positive $(l)$ axons are separated from groups of axons that are unlabeled $(u)$. In $b$, several growth cones (*) are situated next to each other. The 2 largest ones extend off of developing axons and appear to be oriented in opposite directions. Dense LAMP immunoreactivity is evident as punctate deposits along the plasma membranes (arrowheads). The growth cones contain typical agranular reticulum and large accumulations of vesicles $(v)$. These do not appear to be specifically stained. Scale bar: $a, 1.5 \mu \mathrm{m} ; b, 0.5 \mu \mathrm{m}$.

onstrated that LAMP staining in fiber tracts is transient (Horton and Levitt, 1986). Thus, LAMP staining is undetectable in most pathways by the end of the third postnatal week. As noted above, LAMP immunoreactivity is not present on mature axons in vivo (Levitt et al., 1986). A slot-blot assay of fresh, unfixed tissue was performed to determine whether the absence of staining represents a true loss of LAMP expression during development or simply a change in antigen accessibility or masking due to myelination. There have been reports in the literature in which immunoreactivity in aldehyde-fixed tissue sections disappeared in a specific structure, yet assays on fresh tissue revealed that the antigen was still present in the brain, suggesting that fixation can cause technical artifacts leading to misinterpretations of data (Pigott and Kelly, 1986). The results in the present study demonstrate that at P10 the fimbria/fornix and corpus callosum contain high levels of LAMP immunoreactivity, comparable to the hippocampus (Fig. 7). These same tracts, dissected from adults, do not express detectable levels of LAMP in this assay system. Even when the total amount of protein of the adult corpus callosum or fimbria/fornix sample is increased 5- to 10fold, the results are identical, suggesting that the absence of staining that we observed in the brain sections from adults represents a major change in expression of LAMP during axon maturation.

\section{Discussion}

The present immunocytochemical analysis of developing rat brain demonstrates that LAMP is expressed in specific cellular regions and fiber tracts, many of which correspond to structures comprising the limbic system. We found that the distribution in the fetal brain is similar to that exhibited in adults (Levitt, 1984; Levitt et al., 1986), except that axons express immuno- 


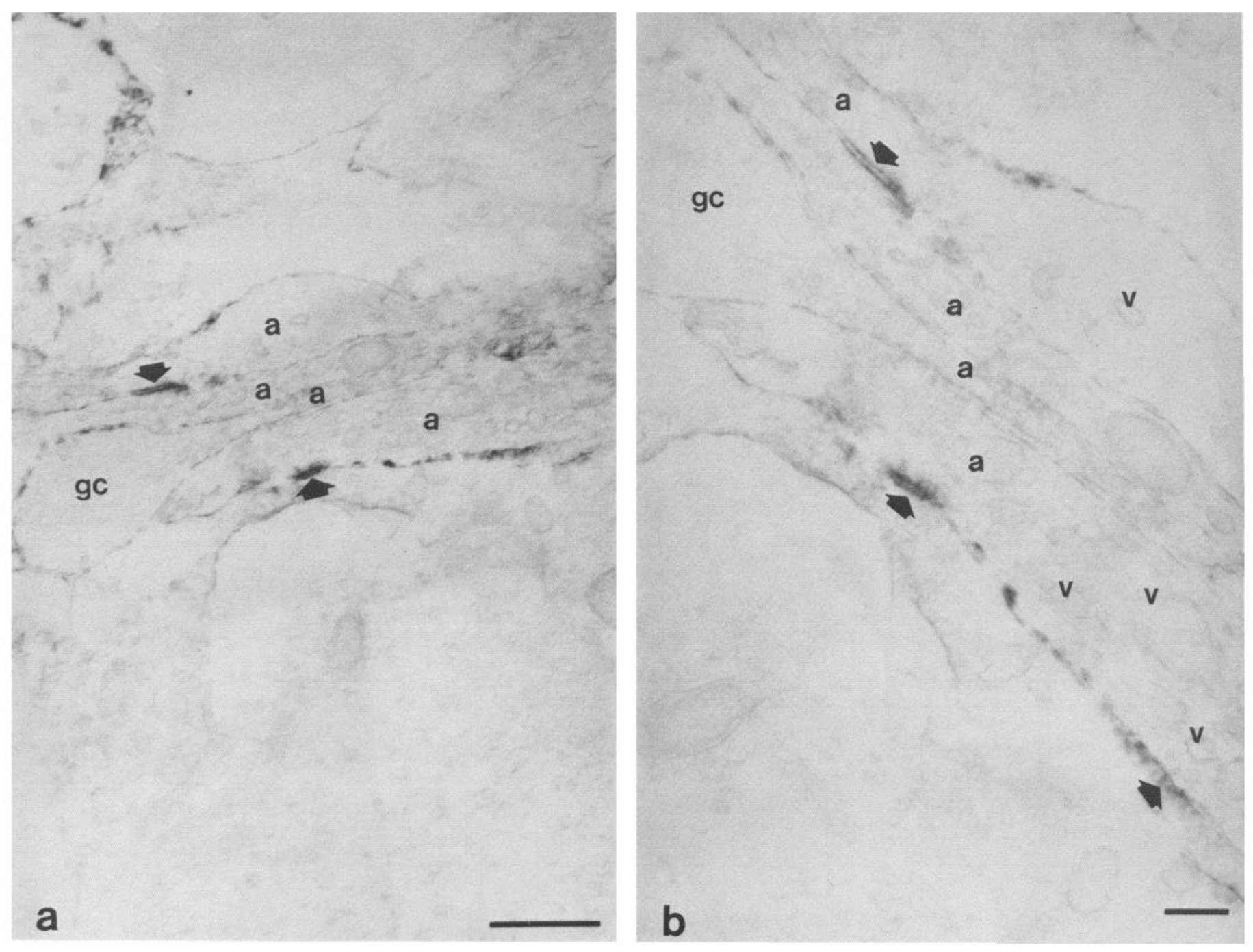

Figure 5. Electron microscopic photomicrographs depicting the surface localization of LAMP on fasciculating axons in the internal capsule at E19. In $a$, spotlike immunoreactivity (black arrows) is present on several axonal processes $(a)$ and their growth cones $(g c)$. Scale bar, $1 \mu \mathrm{m}$. The same field is shown at higher magnification in $b$. In some instances, the immunoreactivity is present as discrete patches on apposing membranes (open arrows) of tightly fasciculated axons. Note the absence of LAMP immunoreactivity on large vesicles within the developing axons and growth cones. Scale bar, $0.5 \mu \mathrm{m}$.

reactivity. LAMP is present in key limbic forebrain structures and in tracts that carry their axons, while it is absent in most regions and tracts that do not form part of the limbic circuitry. Although the specific anatomical distribution is not sufficient to assign LAMP a key role in development, it is consistent with our recent functional studies in which the monoclonal antibody against LAMP was found to prevent the formation of the septohippocampal pathway in vitro (see discussion below).

\section{Specificity of LAMP immunoreactivity in axon tracts}

The present study demonstrates that LAMP-immunorective axons form discrete subsets within developing tracts that carry fiber populations projecting to classically defined limbic system regions. At the light microscopic level, LAMP-immunoreactive axons are present in stereotyped patterns within tracts such as the internal capsule that carry both limbic and nonlimbic axons. Preliminary studies in the fetal cat brain reveal a similar, restricted pattern of LAMP expression within limbic pathways and cellular regions. In addition, the expression of LAMP on fiber tracts is transient. We found, using both immunocytochemical methods on fixed tissue and an immunoassay on fresh, unfixed material, that axonal immunoreactivity is absent by late postnatal ages and remains negative into adulthood. LAMP, however, continues to be expressed on neuronal somata and their dendrites (Levitt et al., 1986). The early, restricted and phylogenetically conserved expression of LAMP demonstrates that developing pathways and cellular regions in the mammalian CNS can exhibit remarkable surface specificity, just as in the invertebrate nervous system (Macagno et al., 1983; McKay et al., 1983; Goodman et al., 1984; Bastiani et al., 1987). Of course, an advantage of such molecular analysis in simpler systems is that the origin and identity of each axon can be documented and thus correlated with specific patterns of molecular expression. In mammalian brain, the complexity and number of neurons, even during development, makes this analysis impossible. Our data demonstrate several features of LAMP expression in fiber tracts. First, tracts in the forebrain that are known to contain mostly projections to or from limbic system regions (e.g., 

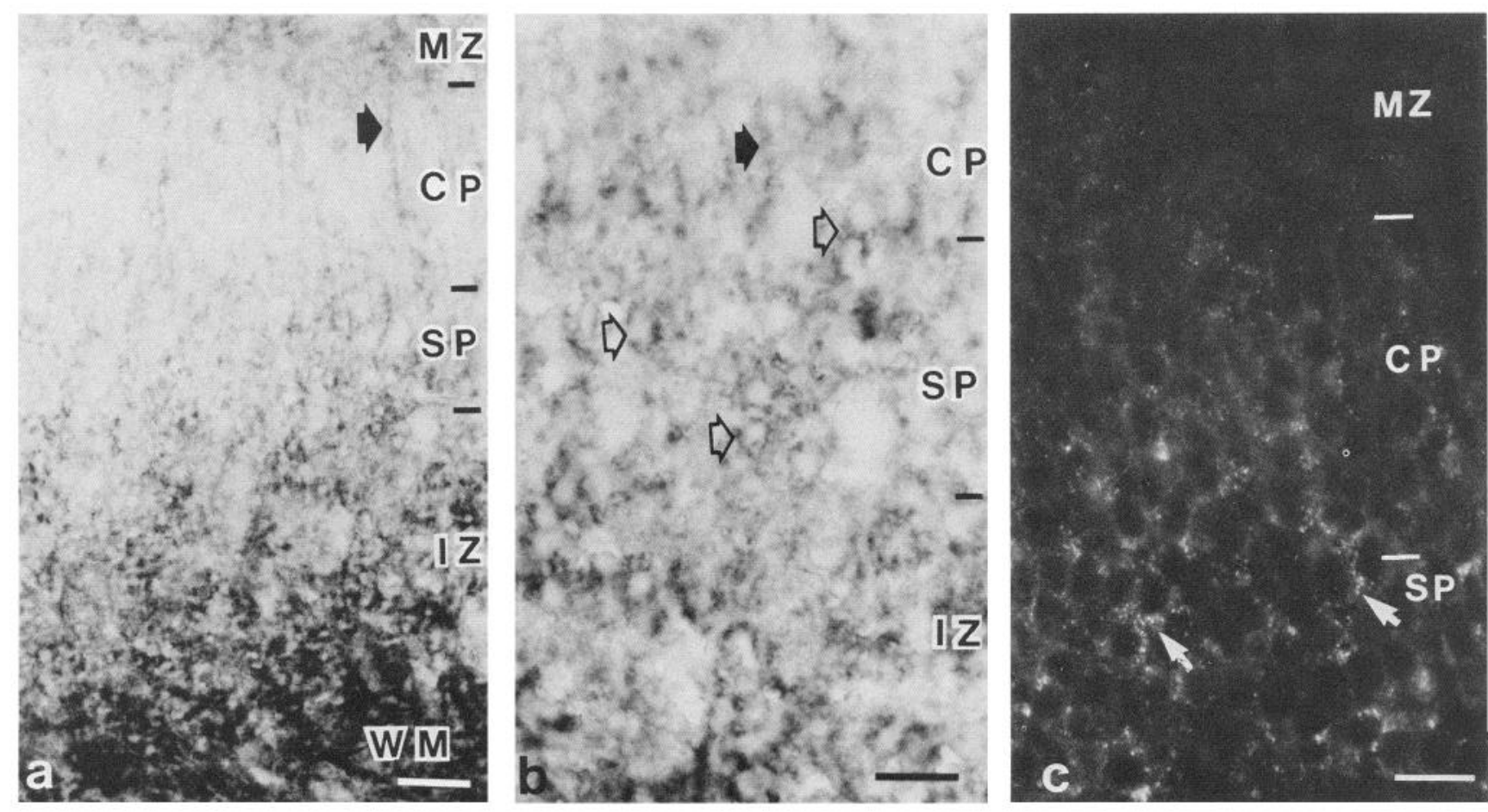

Figure 6. Photomicrographs demonstrating the expression of LAMP immunoreactivity in fetal perirhinal sulcal cortex at E15. $a$, Immunoperoxidase activity displayed throughout the cerebral wall. Most of the cellular staining is seen in the intermediate zone (IZ) and subplate (SP), with a few LAMP-positive cells in the cortical plate $(C P)$. Long, slender profiles also are evident within the CP (arrow) and may represent staining of apical dendrites. $b$, At higher power, one can see LAMP immunoreactivity surrounding cellular profiles (open arrows), as well as process staining (filled arrow). $c$, Anti-LAMP immunofluorescence staining of the surface of cells in the subplate (arrows) and deep cortical plate. In this photograph, staining of the marginal zone is not evident. Scale bar: $a, 25 \mu \mathrm{m} ; b, 10 \mu \mathrm{m} ; c, 15 \mu \mathrm{m}$.

fornix, stria terminalis) express LAMP on most axons, as seen light microscopically. Second, those tracts that contain both limbic-associated and nonlimbic axons (e.g., corpus callosum, anterior commissure, internal capsule) contain only subsets of immunoreactive axons. No study in the mammalian brain has quantified the relative contributions of limbic and nonlimbic axons to a specific fiber pathway comprising both populations, so we can only hypothesize that the unlabeled axons in these tracts form the nonlimbic population. We know, however, that LAMP expression follows topographical organization in these tracts. For example, the caudal part of the corpus callosum, containing axons mostly from the visual cortical regions, is very lightly immunoreactive. This is in contrast to rostral corpus callosum, which contains axons passing between frontal cortices, and is very densely stained with the LAMP antibody. These findings are consistent with our third observation: Tracts carrying nonlimbic system axons, such as the optic nerve, pyramidal tract, medial and lateral lemnisci, and olfactory tracts never exhibit LAMP immunoreactivity at any stage of fetal or postnatal development.

\section{LAMP expression as a marker of limbic neuron differentiation}

Expression of LAMP appears to be related to the time of origin and degree of maturation of neurons. Based on previously published ${ }^{3} \mathrm{H}$-thymidine studies on the time of origin of neurons (Hicks and D'Amato, 1968; Altman and Bayer, 1978; Smart and Smart, 1982), LAMP immunoreactivity is present on septal, thalamic, and cortical (including lateral perirhinal sulcal and medial prefrontal) neurons within $24-36 \mathrm{hr}$ after they undergo their final mitosis. The rapid expression on cortical neurons from the inception of the cortical plate suggests that LAMP is an early marker of neuronal differentiation. This surface antigen, however, does not appear to be expressed on the cortical stem cells in the ventricular zone but is present on cells in the intermediate zone, some of which may be migrating. The expression of the protein occurs relatively early in development, indicating that neurons comprising medial prefrontal and lateral perirhinal sulcal cortex are designated as part of limbic circuitry almost from the time of their initial differentiation. This chemical identity occurs before cytoarchitectonically distinct boundaries are formed and prior to the detectable expression of many neurotransmitters (McDonald et al., 1982; Gall et al., 1984; Miller, 1986; Chun et al., 1987), indicating that LAMP may be involved in events that occur prior to synaptic transmission.

\section{Speculation on the role of LAMP in development}

Our descriptive data only provide supportive evidence for developmental schemes involving chemical specificity in the formation of functional neuronal circuits (Sperry, 1963; Singer et al., 1979; McKay et al., 1983; Bastiani et al., 1987). These results indicate that the findings from developmental studies of simpler nervous systems in the invertebrate are relevant to mammalian brain development. The presence of LAMP on axons and growth cones during pathway formation is similar to the pattern of other antigens found by Bastiani et al. (1987) in the grasshopper and McKay et al. (1983) in the leech. While it has not been demonstrated that the developing mammalian CNS contains pioneer axons that perform initial pathway determination, as described in simpler vertebrates (Kuwada, 1986), the grasshopper and Drosophila (Bentley and Keshishian, 1982; Ho and Goodman, 


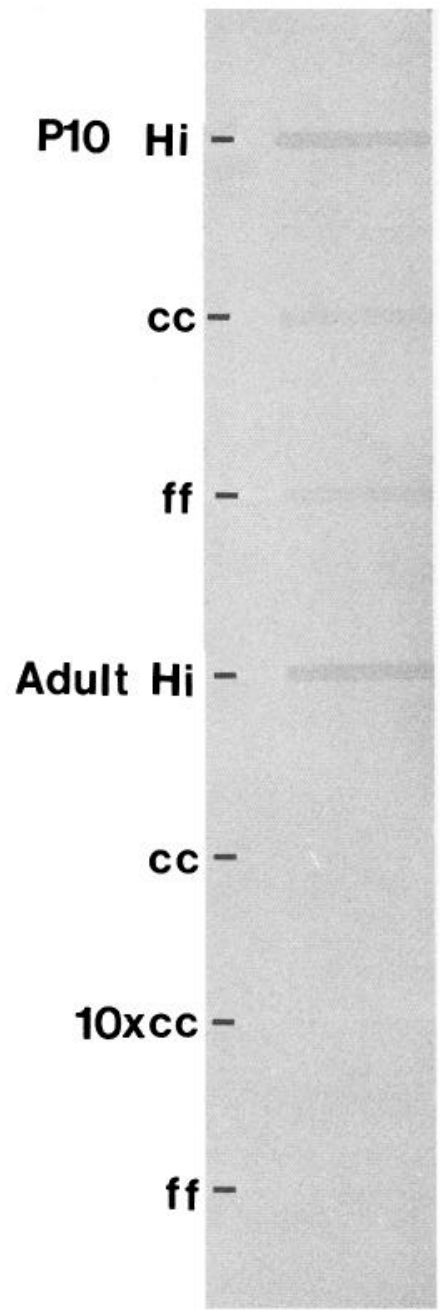

Figure 7. Photograph of a representative slot-blot assay. LAMP immunoreactivity is present in the hippocampus $(h i)$, corpus callosum $(c c)$, and fimbria/fornix $(f)$ in the samples obtained from P10 rats. In the adult, LAMP activity is evident in the hippocampal sample, but absent in the samples of the fiber tracts. In particular, no LAMP activity is evident when total protein applied to the nitrocellulose sheets is increased 10 -fold for the corpus callosum.

1982; Goodman et al., 1984; Blair and Palka, 1985), LAMP is expressed at a time sufficiently early in mammalian brain development to participate, at the molecular level, in such an event. The presence of LAMP in patches along apposing membranes of fasciculating axons is suggestive of such a role. Clearly, this cell-surface protein could participate in different events not related to neural development, but recent studies in an explant culture system directly implicate LAMP as one of the important molecular components necessary for the formation of proper connections between limbic system regions (Keller and Levitt, 1987; F. Keller, K. Rimvall, and P. Levitt, unpublished observations). We found that the anti-LAMP monoclonal antibody can prevent the innervation of the hippocampus by septal cholinergic axons in explant cocultures, while having no effect on general fiber outgrowth or cholinergic neuron survival. The cholinergic axons, rather than entering and collateralizing within the target hippocampus, grow around the explant. Thus, LAMP does not appear to change the ability of axons to fasciculate or grow on a substrate but only alter the ability of the limbic axons to enter a normal target structure. These data, together with the results from the present anatomical study documenting early and spatially restricted expression of LAMP, suggest that this uniquely distributed surface protein may be an important factor in regulating target innervation by specific afferent fiber groups. However, LAMP is unlikely to be the only factor involved in the development of these connections. In fact, we would suggest that LAMP may be part of a complex group of cell-surface determinants, including nonadhesive elements such as ion channels and receptors for diffusable trophic agents, that are expressed early in development to link the components of functional systems in the developing mammalian CNS.

\section{References}

Altman, J., and S. A. Bayer (1978) Development of the diencephalon in the rat. II. Correlation of the embryonic development of the hypothalamus with the time of origin of its neurons. J. Comp. Neurol. 182: 973-994.

Bastiani, M. J., A. L. Harrelson, P. M. Snow, and C. S. Goodman (1987) Expression of fasciclin I and II glycoproteins on subsets of axon pathways during neuronal development in the grasshopper. Cell 48: 745-755.

Bentley, D., and H. Keshishian (1982) Pathfinding by peripheral pioneer neurons in grasshoppers. Science 218: 1082-1088.

Blair, S. S., and J. Palka (1985) Axon guidance in cultured wing discs and disc fragments of Drosophila. Dev. Biol. 108: 411-419.

Chun, J. J. M., M. J. Nakamura, and C. H. Shatz (1987) Transient cells of the developing mammalian cortex are peptide-immunoreactive neurons. Nature 325: 617-620.

Crandall, J. E., and V.S. Caviness (1984) Thalamocortical connections in newborn mice. J. Comp. Neurol. 228: 542-556.

Dodd, J., and T. M. Jessell (1985) Lactoseries carbohydrates specify subsets of dorsal root ganglion neurons projecting to the superficial dorsal horn of rat spinal cord. J. Neurosci. 5: 3278-3294.

Dodd, J., D. Solter, and T. M. Jessell (1984) Monoclonal antibodies against carbohydrate differentiation antigens identify subsets of primary sensory neurons. Nature $311: 469-472$.

Domesick, V. B. (1972) Thalamic relationships of medial cortex in rat. Brain Behav. Evol. 6: 457-483.

Foster, G. A., M. Schultzberg, T. Hökfelt, M. Goldstein, H. C. Hemmings, Jr., C. C. Ouimet, S. I. Walaas, and P. Greengard (1987) Development of a dopamine- and cyclic adenosine $3^{\prime}: 5^{\prime}$-monophosphate-regulated phosphoprotein (DARPP-32) in the prenatal rat central nervous system, and its relationship to the arrival of presumptive dopaminergic innervation. J. Neurosci. 7: 1994-2018.

Gall, C., N. Brecha, K. J. Chang, and H. J. Karten (1984) Ontogeny of enkephalin-like immunoreactivity in the rat hippocampus. Neuroscience 11: 359-380.

Goodman, C. S., M. J. Bastiani, C. Q. Doe, S. du Lac, S. L. Helfand, J. Y. Kuwada, and J. B. Thomas (1984) Cell recognition during neuronal development. Science 225: 1271-1279.

Hawkes, R., E. Niday, and J. Gordon (1982) A dot-immunobinding assay for monoclonal and other antibodies. Anal. Biochem. 119: 142147.

Hendry, S. H. C., E. G. Jones, S. Hockfield, and R. D. G. McKay (1988) Neuronal populations stained with the monoclonal antibody Cat-301 in the mammalian cerebral cortex and thalamus. J. Neurosci. 8: 518542.

Hicks, S. P., and C. J. D'Amato (1968) Cell migrations to the isocortex in the rat. J. Anat. 134: 273-298.

Ho, R. K., and C. S. Goodman (1982) Peripheral pathways are pioneered by an array of central and peripheral neurones in grasshopper embryos. Nature 279: 404-406.

Hockfield, S., R. D. McKay, S. H. C. Hendry, and E. G. Jones (1983) A surface antigen that identifies ocular dominance columns in the visual cortex and laminar features of the lateral geniculate nucleus. Cold Harbor Symp. Quant. Biol. 18: 877-889.

Horton, H. L., and P. Levitt (1986) Developmental studies with a specific cell surface marker for limbic system neurons. In Progress in Developmental Biology, Part A, H. C. Slavkin, ed., pp. 111-113, Liss, New York.

Keller, F., and P. Levitt (1987) Developmental and growth-associated 
regulation of the limbic system associated membrane protein in brain explant cultures. Soc. Neurosci. Abstr. 13: 1145.

Kuwada, J. Y. (1986) Cell recognition by neuronal growth cones in a simple vertebrate embryo. Science 233: 740-746.

Levitt, P. (1984) A monoclonal antibody to limbic system neurons. Science 223: 299-301.

Levitt, P., E. Pawlak-Byczkowska, H. L. Horton, and V. Cooper (1986) Assembly of functional systems in the brain: Molecular and anatomical studies of the limbic system. In The Neurobiology of Down Syndrome, C. J. Epstein, ed., pp. 195-209, Raven, New York.

Lowry, O. H., N. J. Rosebrough, L. Farr, and R. J. Randall (1951) Protein measurement with the folin phenol reagent. J. Biol. Chem. 193: 265-275.

Loy, R., G. Lynch, and C. W. Cotman (1977) Development of afferent lamination in the fascia dentata of the rat. Brain Res. 121: 229-243.

Macagno, E. R., R. R. Stewart, and B. Zipser (1983) The expression of antigens by embryonic neurons and glia in segmental ganglia of the leech Haemopsi marmarata. J. Neurosci. 3: 1746-1759.

McDonald, J. K., J. G. Parnavelas, A. N. Karamanlidis, and N. J. Brecha (1982) The morphology and distribution of peptide-containing neurons in the adult and developing visual cortex of the rat. II. Vasoactive intestinal peptide. J. Neurocytol. 11:825-837.

McKay, R. D. G., S. Hockfield, J. Johanson, I. Thompson, and K. Frederickson (1983) Surface molecules identify groups of growing axons. Science 222: 788-794.

Miller, M. W. (1986) The migration and neurochemical differentiation of $\gamma$-aminobutyric acid (GABA)-immunoreactive neurons in rat visual cortex as demonstrated by a combined immunocytochemicalautoradiographic technique. Dev. Brain. Res. 28: 41-46.

Papez, J. W. (1937) A proposed mechanism of emotion. Arch. Neurol. Psychol. 38: 725-743.

Pawlak-Byczkowska, E., and P. Levitt (1985) Unique nucleus-associated proteins in different cell types in the developing and adult brain revealed by monoclonal antibodies. Soc. Neurosci. Abstr. 11: 1212.

Penfield, W., and B. Milner (1958) Memory deficit produced by bilateral lesions in the hippocampal zone. Arch. Neurol. Psychol. 79: 475-497.

Pigott, R., and J. S. Kelly (1986) Immunohistochemical and biochemical studies with the monoclonal antibody 69A1: Similarities of the antigen with cell adhesion molecules L1, NILE and Ng-CAM. Dev. Brain Res. 29: 111-122.

Platt, J. L., and A. F. Michael (1983) Retardation of fading and enhancement of intensity of immunofluorescence by $p$-phenylenediamine. J. Histochem. Cytochem. 31: 840-842.

Purves, D., and J. W. Lichtman (1985) Principles of Neural Development, Sinauer, Sunderland, MA

Rakic, P. (1974) Neurons in rhesus monkey visual cortex: Systematic relation between time of origin and eventual disposition. Science 183 : 425-427.

Rakic, P. (1976) Prenatal genesis of connections subserving ocular dominance in the rhesus monkey. Nature 261: 467-471.

Scoville, W., and B. Milner (1957) I oss of recent memory after bilateral hippocampal lesions. J. Neurol. Neurosurg. Psychiatry 20:1121.

Singer, M., R. H. Nordlander, and M. Egar (1979) Axonal guidance during embryogenesis and regeneration in the spinal cord of the newt: The blueprint hypothesis of neuronal pathway patterning. J. Comp. Neurol. 185: 1-22.

Smart, I. H. M., and M. J. Smart (1982) Growth patterns in the lateral wall of the mouse telencephalon. I. Autoradiographic studies of the histogenesis of the isocortex and adjacent areas. J. Anat. 134: 273298.

Sperry, R. W. (1963) Chemoaffinity in the orderly growth of nerve fiber patterns and connections. Proc. Natl. Acad. Sci. USA 50: 703710.

Trisler, G., J. Bekenstein, and M. P. Daniels (1986) Antibody to a molecular marker of cell position inhibits synapse formation in retina. Proc. Natl. Acad. Sci. USA 83: 4194-4198.

Trisler, G., M. D. Schneider, and M. Nirenberg (1981) A topographic gradient of molecules in retina can be used to identify neuron position. Proc. Natl. Acad. Sci. USA 78: 2145-2149.

Valentino, K. L., and E. G. Jones (1982) The early formation of the corpus callosum: A light and electron microscopic study in foetal and neonatal rats. J. Neurocytol. 11: 583-609.

Wyss, J. M., L. W. Swanson, and W. M. Cowan (1979) A study of subcortical afferents to the hippocampal formation. Neuroscience 4 : $463-476$. 